Article

\title{
Developing a Microbial Consortium for Enhanced Metabolite Production from Simulated Food Waste
}

\author{
Nathan D. Schwalm III 1,*®D, Wais Mojadedi ${ }^{2}$, Elliot S. Gerlach ${ }^{1}$, Marcus Benyamin ${ }^{2}$, \\ Matthew A. Perisin ${ }^{1}$ and Katherine L. Akingbade ${ }^{1, *}$ \\ 1 Combat Capabilities Development Command Army Research Laboratory, FCDD-RLS-EB, 2800 Powder Mill \\ Road, Adelphi, MD 20783, USA; elliot.s.gerlach.civ@mail.mil (E.S.G.); \\ matthew.a.perisin.civ@mail.mil (M.A.P.) \\ 2 Oak Ridge Associated Universities, 4692 Millennium Drive, Suite 101, Belcamp, MD 21017, USA; \\ wais.mojadedi.ctr@mail.mil (W.M.); marcus.s.benyamin2.ctr@mail.mil (M.B.) \\ * Correspondence: nathan.d.schwalm.civ@mail.mil (N.D.S.III); katherine.l.akingbade.civ@mail.mil (K.L.A.); \\ Tel.: +301-394-0193 (N.D.S.III); +301-394-0604 (K.L.A.)
}

Received: 25 September 2019; Accepted: 22 November 2019; Published: 27 November 2019

\begin{abstract}
Food waste disposal and transportation of commodity chemicals to the point-of-need are substantial challenges in military environments. Here, we propose addressing these challenges via the design of a microbial consortium for the fermentation of food waste to hydrogen. First, we simulated the exchange metabolic fluxes of monocultures and pairwise co-cultures using genome-scale metabolic models on a food waste proxy. We identified that one of the top hydrogen producing co-cultures comprised Clostridium beijerinckii NCIMB 8052 and Yokenella regensburgei ATCC 43003. A consortium of these two strains produced a similar amount of hydrogen gas and increased butyrate compared to the $C$. beijerinckii monoculture, when grown on an artificial garbage slurry. Increased butyrate production in the consortium can be attributed to cross-feeding of lactate produced by $Y$. regensburgei. Moreover, exogenous lactate promotes the growth of $C$. beijerinckii with or without a limited amount of glucose. Increasing the scale of the consortium fermentation proved challenging, as two distinct attempts to scale-up the enhanced butyrate production resulted in different metabolic profiles than observed in smaller scale fermentations. Though the genome-scale metabolic model simulations provided a useful starting point for the design of microbial consortia to generate value-added products from waste materials, further model refinements based on experimental results are required for more robust predictions.
\end{abstract}

Keywords: butyrate; Clostridium beijerinckii; cross-feeding; food waste; genome-scale metabolic model; hydrogen; lactate; Yokenella regensburgei

\section{Introduction}

Generation of food waste is a global problem [1,2]. Approximately $33 \%$ of food produced is wasted, contributing to 30-60\% of the total solid waste produced worldwide [3]. The burden of food waste disposal extends to military environments, where it imposes an additional logistical burden on Army operations [4]. The primary waste produced by the soldier is food related trash [4], and current practices dictate that field generated waste must either be buried or burned daily [5]. An alternative to these practices is the anaerobic fermentation of food waste to commodity chemicals [1]. Anaerobic fermentation of a single carbon feedstock is frequently used in industrial-scale processes to produce biofuels [6] and commodity chemicals [7]. Significant challenges exist applying anaerobic fermentations to food waste breakdown, due to the complexity and inconsistency of the fermentation feedstock. Utilizing additional microbes that can support the primary fermentation strain as part of a microbial 
consortium is a potentially promising avenue to increasing the viability of food waste to value-added chemical conversion.

Considerable research efforts are underway to harness both microbial consortia comprising multiple genotypes of the same species [8,9] and multi-species consortia $[10,11]$ for improved production of chemicals from readily available complex substrates [12,13]. Microbial consortia enable separation of complex substrate breakdown and chemical production between different species, which can reduce the metabolic burden on individual cells and reduce the need to engineer these microbes [14,15]. Cross-feeding of metabolites from extracellular substrate breakdown [16], cellular secretion [17], or via direct cell-to-cell interactions $[18,19]$ can support the stability of a microbial consortium. These interactions can be designed to force the dependence of auxotrophic strains on each other for growth [20] or can be a result of native metabolic byproducts from one organism acting as a substrate for another organism [21].

Hydrogen is a promising alternative fuel source to petrochemicals due to its high energy content (120 MJ/kg versus $46.7 \mathrm{MJ} / \mathrm{kg}$ for gasoline) [22]. Microbes primarily produce hydrogen via photofermentation by the purple non-sulfur bacteria Rhodobacter and Rhodopseudomonas [23], and during dark fermentation by strictly anaerobic Clostridium species [22]. In purple non-sulfur bacteria, hydrogen production is coupled with organic acid utilization [23]. In Clostridium species, hydrogen production is coupled with ferredoxin oxidation and is accompanied by organic acid production [24], which inhibits hydrogenase activity and hydrogen production [25]. Microbial consortia of Clostridium that generate organic acids and purple non-sulfur bacteria that use organic acids for hydrogen production are being explored [26,27], as are consortia comprising multiple Clostridium species [13,28]. The formate-hydrogen lyase complex, found mainly in Proteobacteria, produces hydrogen via the anaerobic breakdown of formate [29]. This complex is also being explored as a route for hydrogen production in Escherichia coli engineered to express a hydrogenase [29], and as part of microbial consortia with Clostridium [30].

Genome scale metabolic models (GSMMs), combined with flux-balance analysis (FBA), provide predictive frameworks not only to assess the metabolic outputs from a variable inputs, but also to assess metabolic interactions between multiple microbes. These models are built from enzymatic genome annotations that determine available reactions for each microbe, resulting in a table of reactants/products by reaction [31]. These tables are then fed to FBA to determine what metabolic outputs are possible given a set of inputs, assuming a steady-state [32]. To further limit the solution space, there is an assumption that each microbe will optimize growth (i.e., biomass production) [32]. This framework has been demonstrated to accurately recapitulate single microbe metabolisms and metabolic interactions between members of a consortium [33-37].

Recently, Magnusdottir et al. created GSMMs of approximately equal refinement for 773 gut microbes and simulated all monocultures and co-cultures on Western and high-fiber diet inputs [38]. Ecological interactions between these microbes were predicted to vary based on the diet and whether the environment was aerobic or anaerobic [38]. Perisin and Sund followed up on these findings to predict whether combinations of microbes could produce specialty/commodity chemicals at higher rates than monocultures [39]. Co-cultures were identified that could overproduce chemicals of interest such as hydrogen gas, butanol, methane, formaldehyde, propionate, and urea [39].

We developed a framework to experimentally test commodity chemical production from microbial consortia predicted to synergistically overproduce an individual chemical (Figure 1). We used genome-scale metabolic models and flux balance analysis of human gut microbiota strains provided a simulated food waste to identify two species bacterial consortia with the greatest synergistic production of hydrogen gas. We then measured the gases and metabolites produced by one of the highest overproducing consortia and its component species when cultured on an artificial garbage slurry medium (AGS). Moreover, we demonstrated the cross-feeding of metabolites between the two species. Finally, we attempted to scale-up the AGS fermentation with two distinct conditions, stationary fermentation or stirred fermentation with $\mathrm{pH}$ control, to assess the feasibility of the 
method. Experimental consortium fermentations will further improve metabolic models for future experimental testing.

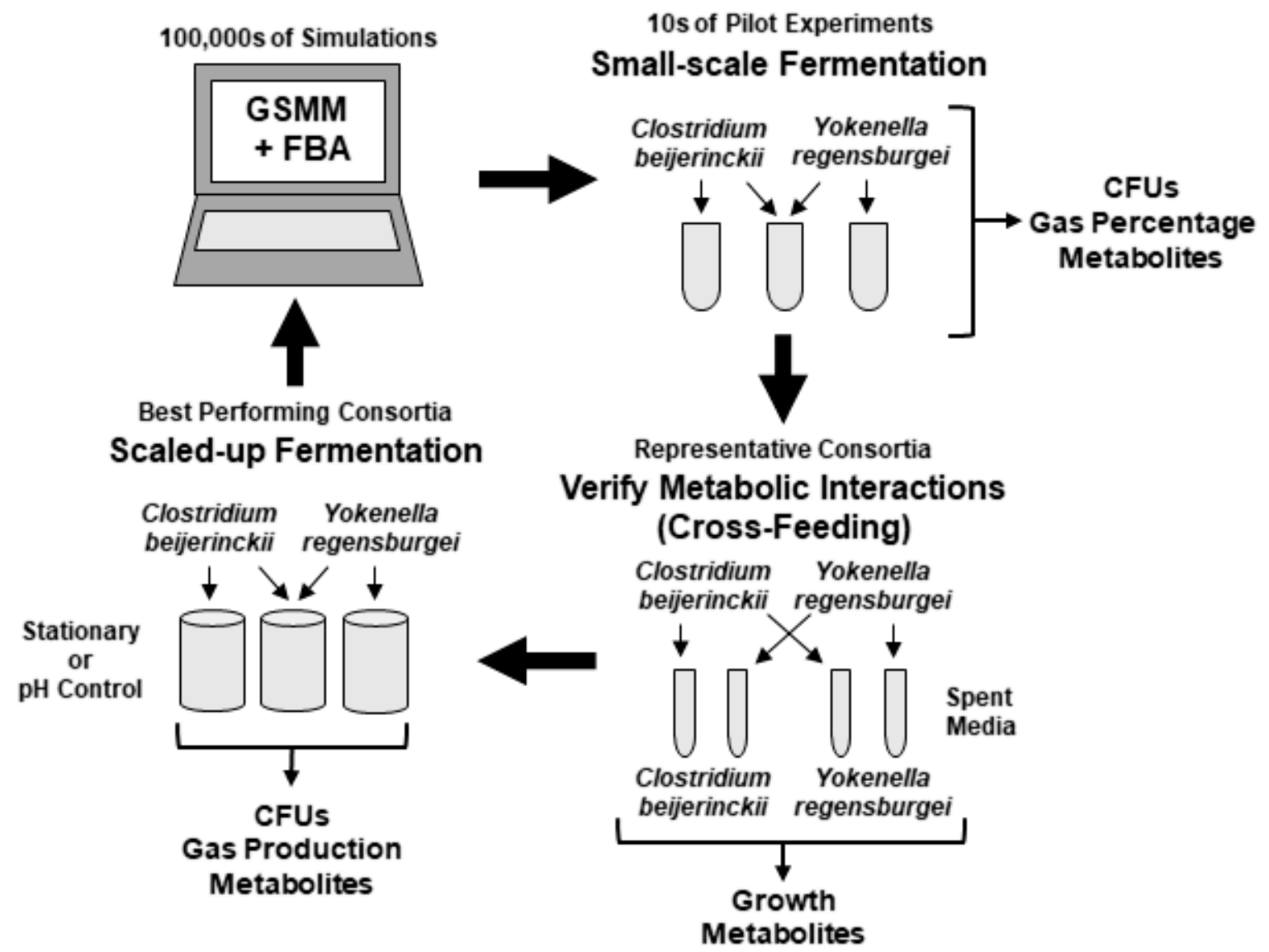

Figure 1. Experimental framework. Genome-scale metabolic modeling (GSMM) and flux balance analysis (FBA) were used to predict the metabolic outputs of 298,378 pairwise combinations of microbial consortia provided a simulated food waste medium. The consortium of Clostridium beijerinckii and Yokenella regensburgei was predicted to have the second highest synergistic overproduction of hydrogen compared to any individual strain. Small-scale fermentations were performed using the C. beijerinckii and $Y$. regensburgei consortium on an artificial garbage slurry medium to simulate food waste. Growth (CFUs), metabolites, and percentage of gases produced in gas chromatography vials were measured. Spent media from cultures of $C$. beijerinckii and Y. regensburgei were used to cross-feed to the other species, and growth and metabolite production were measured. Small-scale experiments were used to inform design of scaled-up fermentations of monocultures and the consortium of C. beijerinckii and Y. regensburgei. The results of these experiments can be used to further improve the GSMM and FBA forming a broad framework for testing production of commodity chemicals by microbial consortia.

\section{Materials and Methods}

\subsection{Genome-Scale Metabolic Modeling}

Simulations were performed as in Perisin and Sund [39]. Genome-scale metabolic models (GSMMs) for Clostridium beijerinckii NCIMB 8052 and Yokenella regensburgei ATCC 43003 (AGORA v 1.01) were downloaded from the Virtual Metabolic Human database (http://vmh.uni.lu) [38]. Lower bounds for input exchange reactions were set to mimic Western diet food waste (Supplementary Table 1 in [39]). All simulations were performed in R v3.4.0 [40] with plots created using ggplot2 v 2.2.1 [41]. Systems Biology Markup Language (SBML) models were first uploaded with the sybilSBML v 3.0.1 [42] package. Then, sybil v2.0.4 [43], and glpkAPI v 1.3.0 [44] packages were used for model manipulations and 
flux-balance analysis (FBA). For monoculture simulations, flux through each model's biomass reaction was maximized. The maximum biomass flux was then used to run parsimonious enzyme usage FBA (pFBA, mtf algorithm in sybil) so that the total absolute flux was minimized. For co-culture simulations, models were combined in a similar manner to Magnusdottir et al. [38]. The COBRA [45] MATLAB script, createMultipleSpeciesModel.m found at: https:/github.com/opencobra/cobratoolbox, which is based on the FBA implementation in Klitgord and Segre [46], was used as a template. This script was converted to work in $\mathrm{R}$ with the sybil package and creates a common environment for metabolites to be exchanged between models, but does not create a host compartment as in the COBRA implementation. After combining the models, input exchange fluxes were updated based on Western diet food waste as above, and pFBA was used to simulate growth, simultaneously maximizing each model's growth and minimizing the total absolute flux.

\subsection{Bacterial Strains and Growth Conditions}

Clostridium beijerinckii NCIMB 8052 (ATCC 51743) and Yokenella regensburgei ATCC 43,003 were obtained from ATCC. Both strains were cultivated in anaerobic conditions (5\% carbon dioxide, $5 \%$ hydrogen, $90 \%$ nitrogen) at $37^{\circ} \mathrm{C}$ in a Coy anaerobic chamber and maintained as stocks at $-80{ }^{\circ} \mathrm{C}$ in media containing 20\% glycerol. C. beijerinckii was routinely cultured on Clostridial Growth Medium (CGM) [47] or Luria Bertani (LB) medium (Fisher, Fair Lawn, NJ, USA), supplemented with 0.5\% glucose, unless otherwise noted. Thiamphenicol $\left(30 \mu \mathrm{g} \mathrm{mL}^{-1}\right)$ was used to select for C. beijerinckii when necessary [48,49]. Y. regensburgei was routinely cultured on LB supplemented with $0.5 \%$ glucose or Brain Heart Infusion (BHI) medium (Oxoid, Lenexa, KS, USA). Erythromycin $\left(40 \mu \mathrm{g} \mathrm{mL}{ }^{-1}\right)$ and aerobic growth at $37^{\circ} \mathrm{C}$ were used to select for $Y$. regensburgei, when necessary [49]. The artificial garbage slurry was generated to model organic solid waste, as previously described [50], with limited modifications. Briefly, 10\% $\left(\mathrm{w} \mathrm{v}^{-1}\right)$ dog food (Kibble 'n Bits ${ }^{\circledR}$ Chef Choice Bistro Oven Roasted Beef, Spring Vegetable \& Apple Flavor) was blended in distilled water and autoclaved $\left(121{ }^{\circ} \mathrm{C}, 30 \mathrm{~min}\right.$ exposure). Following the first autoclave cycle, the $\mathrm{pH}$ was balanced to 7.0 via addition of sodium hydroxide solution and buffered to $50 \mathrm{mM}$ potassium phosphate $\mathrm{pH}$ 6.7, prior to a second autoclave cycle. For cross-feeding experiments, C. beijerinckii or Y. regensburgei were grown in LB supplemented with $0.5 \%$ glucose for $24 \mathrm{~h}$ and filtered through a $0.2 \mu \mathrm{m}$ PES membrane (Corning, Corning, NY, USA) to produce spent media. Spent media with the addition of $50 \mathrm{mM}$ potassium phosphate $\mathrm{pH} 6.8$ was used for an additional $24 \mathrm{~h}$ of culturing of either C. beijerinckii or $Y$. regensburgei. For experiments assessing the ability of $C$. beijerinckii to utilize exogenous lactate, the indicated amount of DL-lactic acid (Fluka, St. Louis, MO, USA) was added to cultures and neutralized with sodium hydroxide. Bacterial growth was assessed by measuring absorbance at $600 \mathrm{~nm}$, for cross-feeding and lactate supplementation experiments, or plating of multiple dilutions to count colony forming units per milliliter $\left(\mathrm{CFU} \mathrm{mL}^{-1}\right)$ on selective media, for experiments using AGS.

\subsection{Bioreactor Fermentations}

Bioreactor fermentations were conducted in a DASGIP ${ }^{\circledR}$ parallel fermentation system (Eppendorf, Jülich, Germany). AGS was brought to $\mathrm{pH} 6.7$ by addition of sodium hydroxide solution, following the addition of the $50 \mathrm{mM}$ potassium phosphate and aliquoted to reaction vessels prior to autoclaving $\left(121^{\circ} \mathrm{C}, 30 \mathrm{~min}\right.$ exposure) a single time. For the $\mathrm{pH}$-controlled bioreactor experiment, the DASGIP ${ }^{\circledR}$ system automatically added sodium hydroxide to maintain a $\mathrm{pH}$ of at least 6.7. Temperature was maintained at $37{ }^{\circ} \mathrm{C}$ and anaerobic headspace maintained via nitrogen addition at a flow rate of $1 \mathrm{~L} \mathrm{~min}^{-1}$.

\subsection{HPLC Quantification of Metabolites}

Fermentation products were separated and quantified using an Aminex HPX-87H organic acid column $(300 \times 7.8 \mathrm{~mm}$; Bio-Rad, Hercules, CA, USA) on an Agilent 1200 series HPLC (Agilent Technologies, Santa Clara, CA, USA) equipped with a multi-wavelength UV/vis detector $(210 \mathrm{~nm}$ 
and $280 \mathrm{~nm}$ ) and refractive index detector. Samples were filtered through a $0.2 \mu \mathrm{m}$ PES membrane (Corning) and stored at $4{ }^{\circ} \mathrm{C}$ until applied to the column with an injection volume of $20 \mu \mathrm{L}$. Separation was performed using an isocratic mobile phase consisting of $3.25 \mathrm{mM}$ sulfuric acid at a flow rate of $0.600 \mathrm{~mL} \mathrm{~min}^{-1}$ for a total run time of $55 \mathrm{~min}$, and was temperature controlled for the entire duration at $35^{\circ} \mathrm{C}$. Quantification of acetate, acetone, butyrate, ethanol, formate, glucose, and lactate was performed through the use of standardized concentration gradients with HPLC-grade standards obtained from various vendors (not shown). Data was processed using ChemStation (Agilent).

\subsection{Gas Chromatography}

Hydrogen and carbon dioxide gas production were measured using a gas chromatograph (GC; Infinicon 3000 microGC ${ }^{\circledR}$ ) equipped with a thermal conductivity detector. A fixed molecular sieve 5A capillary column using argon as the carrier gas was used for hydrogen and nitrogen quantification, and a Plot U column with helium as the carrier gas was used for carbon dioxide quantification. GC data was processed using ezIQ and Diablo EZReporter software (Diablo Analytical, Antioch, CA, USA). For experiments performed in the anaerobic chamber, immediately after inoculation, $1.5 \mathrm{~mL}$ of each culture was transferred to a GC vial that was then sealed. For bioreactor sampling, the same parameters were utilized with samples automatically taken from the headspace every $11 \mathrm{~min}$ via an in-line setup. Gas production rates were derived from the GC gas percentage output using the ideal gas law, headspace flowrate $\left(1 \mathrm{~L} \mathrm{~min}^{-1}\right)$, and the fermentation volume $(1 \mathrm{~L})$. The trapezoidal rule was used to approximate total gas production from the GC production rates using numerical integration.

\section{Results}

\subsection{Co-culture Simulations Predict Higher Hydrogen Production than Both Monocultures}

A consortium of C. beijerinckii NCIMB 8052 and Y. regensburgei ATCC 43,003 was originally identified as having the second highest predicted hydrogen production among 298,378 simulated co-cultures (Table 1) [39]. These co-culture simulations represented every pairwise combination of 773 human gut microbe GSMMs [38]. Upon further examination of output exchange fluxes for the co-culture of $C$. beijerinckii and $Y$. regensburgei and their monocultures, C. beijerinckii was predicted to be the sole hydrogen producer (Figure 2). Compared to the monoculture, the addition of $Y$. regensburgei boosted C. beijerinckii biomass (Figure 2) and hydrogen production (Figure 2). The increased hydrogen production was not due only to increased biomass, because when the hydrogen flux was normalized to growth rate, the co-culture hydrogen production rate $(86$ millimoles per gram dry cell weight per hour) was greater than the monoculture hydrogen production (69 millimoles per gram dry cell weight per hour). The increase in co-culture output fluxes were predicted to be due to cross-feeding of lactate from $Y$. regensburgei to $C$. beijerinckii (Figure 2). The overall ecological interaction was predicted to be commensal as the growth rate of $C$. beijerinckii increased from the monoculture to the co-culture, while the growth rate $Y$. regensburgei was unchanged(Figure 2). Additional microbial consortia containing C. beijerinckii that were predicted to have more than two-fold greater hydrogen production than $C$. beijerinckii alone are listed in Table 1. 
Table 1. Microbial consortia predicted to have greater than two-fold C. beijerinckii hydrogen flux. Organisms predicted to increase the hydrogen flux greater than two-fold over the hydrogen flux of $C$. beijerinckii alone, when simulated in a co-culture with $C$. beijerinckii. Changes in predicted biomass for each organism ( $\triangle$ Biomass Organism) and the predicted biomass of $C$. beijerinckii ( $\Delta$ Biomass $C$. beijerinckii) from simulated co-cultures versus simulated monocultures.

\begin{tabular}{cccc}
\hline Organism & $\begin{array}{c}\text { Fold Hydrogen versus } \\
\text { C. Beijerinckii Alone }\end{array}$ & $\begin{array}{c}\Delta \text { Biomass } \\
\text { C. Beijerinckii }\end{array}$ & $\begin{array}{c}\Delta \text { Biomass } \\
\text { Organism }\end{array}$ \\
\hline Yokenella regensburgei ATCC 43003 & 2.25 & 0.58 & 0 \\
Kluyvera ascorbata ATCC 33433 & 2.20 & 0.54 & 0 \\
Hafnia alvei ATCC 51873 & 2.08 & 0.48 & 0 \\
Yersinia rohdei ATCC 43380 & 2.07 & 0.49 & 0 \\
Yersinia kristensenii ATCC 33638 & 2.07 & 0.49 & 0 \\
Vibrio mimicus MB 451 & 2.06 & 0.43 & 0 \\
Solobacterium moorei DSM 22971 & 2.06 & -0.34 & 0.51 \\
Escherichia hermannii NBRC 105704 & 2.05 & 0.44 & 0 \\
Vibrio parahaemolyticus RIMD 2210633 & 2.05 & 0.45 & 0.21 \\
Capnocytophaga sputigena ATCC 33612 & 2.05 & -0.29 & 0.43 \\
Lactococcus garvieae ATCC 49156 & 2.05 & 0.42 & 0 \\
Trabulsiella guamensis ATCC 49490 & 2.01 & 0.37 & 0.28 \\
Cellulosimicrobium cellulans J36 & 2.01 &
\end{tabular}
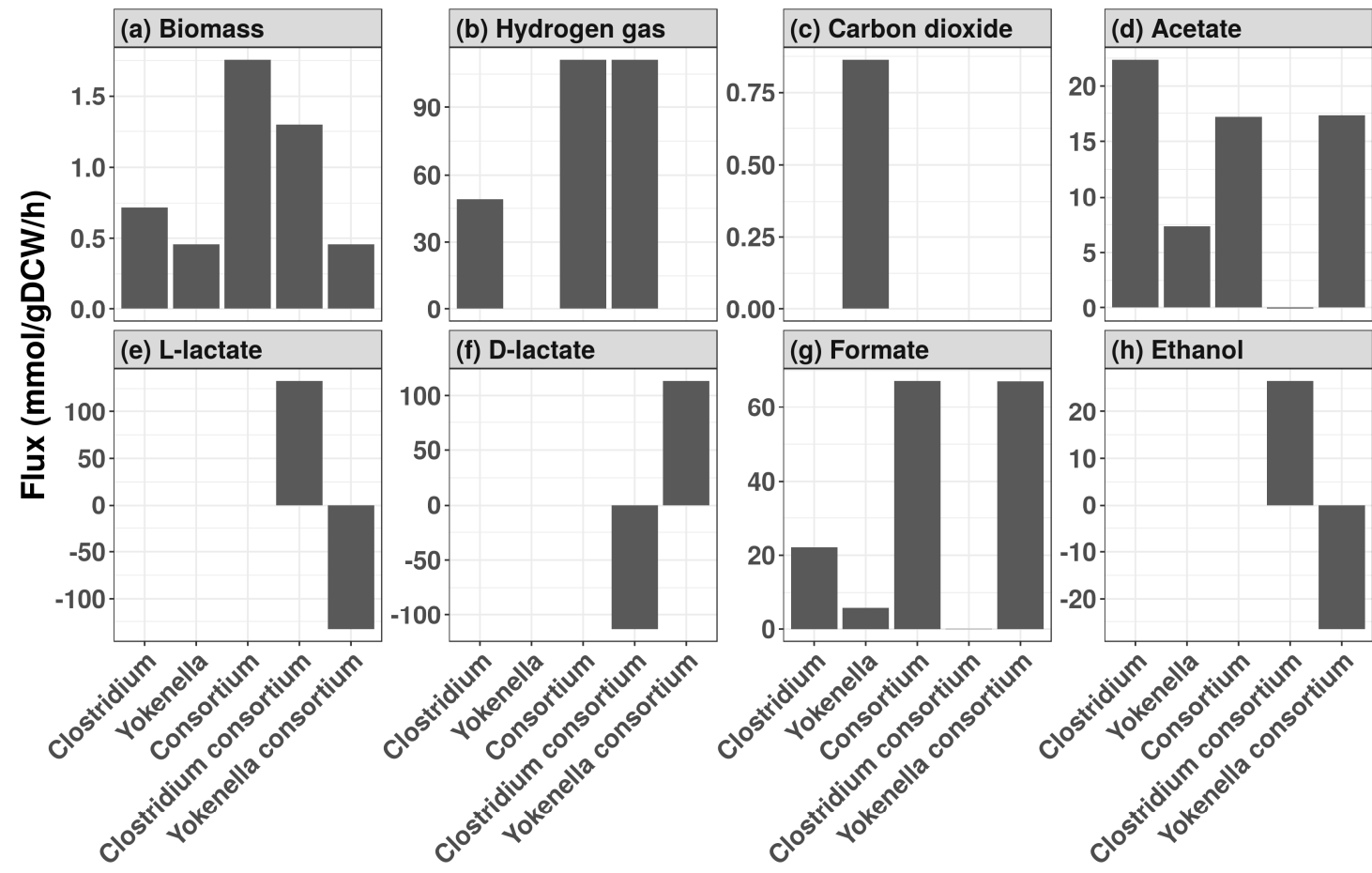

\section{Condition}

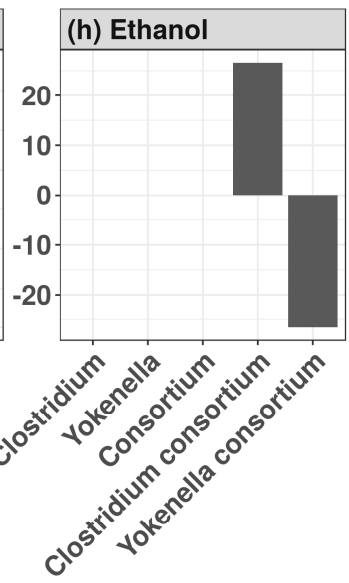

Figure 2. Predicted metabolite flux for a consortium of $C$. beijerinckii and Y. regensburgei. Flux balance analysis (FBA) simulations of Clostridium beijerinckii and Yokenella regensburgei monocultures (Alone), and co-cultures of $C$. beijerinckii and $Y$. regensburgei (Consortium) to predict production of (a) biomass, (b) hydrogen, (c) carbon dioxide, (d) acetate, (e) L-lactate, (f) D-lactate, (g) formate, (h) ethanol. C. beijerinckii Consortium indicates the contribution of $\mathrm{C}$. beijerinckii to the metabolites in the co-culture FBA and $Y$. regensburgei Consortium indicates the contribution of $Y$. regensburgei to the metabolites in the co-culture FBA. Units of simulated flux are millimoles of metabolite produced per gram of dry cell weight per hour. 


\subsection{Metabolite Production by a Consortium of C. beijerinckii and Y. regensburgei}

The consortium of C. beijerinckii NCIMB 8052 and Y. regensburgei ATCC 43,003 (Table 1) was predicted to exhibit the second largest increase in hydrogen production over any individual modeled bacterial strain when provided with a food waste simulant. To test for the potential of this interaction in vivo, cells were grown on an artificial garbage slurry medium (AGS) in an anaerobic chamber. After $24 \mathrm{~h}$ growth, $C$. beijerinckii and $Y$. regensburgei were plated on selective media to determine the number of colony forming units per milliliter of culture (CFU mL ${ }^{-1}$ ) for each strain. We observed an increase in CFU mL ${ }^{-1}$ over the inoculum for both strains, indicating that AGS supports the growth of these bacteria independently (Figure 3a). Both strains also grew within $24 \mathrm{~h}$ in a co-culture, as measured by plating for CFUs on selective media (Figure 3a). The CFU count of each strain in the consortia was significantly lower than for each individual strain (Figure 3a). However, the total CFU count of both strains in the consortia was higher than for the cultures of $C$. beijerinckii alone and similar to the CFU count in the $Y$. regensburgei monocultures (Figure 3a). Viable cells were unable to be consistently recovered by plating for CFUs at subsequent times (data not shown), likely due to depletion of an essential growth factor or accumulation of a toxic byproduct in the media.

We measured gas production by C. beijerinckii and Y. regensburgei, either alone or as part of a consortium, as an increase in atmospheric percentage over the background atmosphere by gas chromatography (GC), after three days of growth on the artificial garbage slurry medium in sealed vials. C. beijerinckii produced approximately $8 \%$ hydrogen above the atmospheric background, whereas $Y$. regensburgei produced only about $1 \%$ hydrogen above the atmospheric background, when each was cultured individually (Figure $3 b$ ). The consortium produced a statistically similar percentage of hydrogen above the background ( $p=0.89$, by two-tailed Student's $t$ test) to the C. beijerinckii monoculture (Figure $3 b$ ). Each individual strain and the consortium of the two strains produced an approximately equivalent proportional percentage of carbon dioxide to hydrogen (Figure 3b).

We quantified the organic acids produced by C. beijerinckii and Y. regensburgei individually and as a consortium from filtered extracts via HPLC. Both C. beijerinckii monocultures and the consortium primarily produced butyrate, consistent with previous reports [28], with the consortium producing a small but significantly larger amount than the $C$. beijerinckii monoculture (Figure 3c). As predicted by the FBA (Figure 2), the Y. regensburgei monoculture produced almost no butyrate (Figure 3c), but produced approximately $30 \mathrm{mM}$ lactate, which was not observed in either the consortium or the C. beijerinckii monoculture (Figure 3d). The consortium produced substantially lower lactate than would be expected, if the $Y$. regensburgei present produced a proportional amount of lactate to that in the $Y$. regensburgei monoculture (Figure $3 \mathrm{~d}$ ). Acetate was a secondary fermentation product in all cultures, accumulating to approximately $11 \mathrm{mM}$ in both the $C$. beijerinckii monoculture and the consortium, and approximately $4 \mathrm{mM}$ in the Y. regensburgei monoculture (Figure 3e). A significant amount of formate was consumed in both monocultures, while the consortium produced a small amount of formate (Figure 3f). The consumption of formate by $Y$. regensburgei could be an indication of formate-hydrogen lyase activity [29], although low amounts of hydrogen were observed for $Y$. regensburgei monocultures (Figure 3b). 
(a)

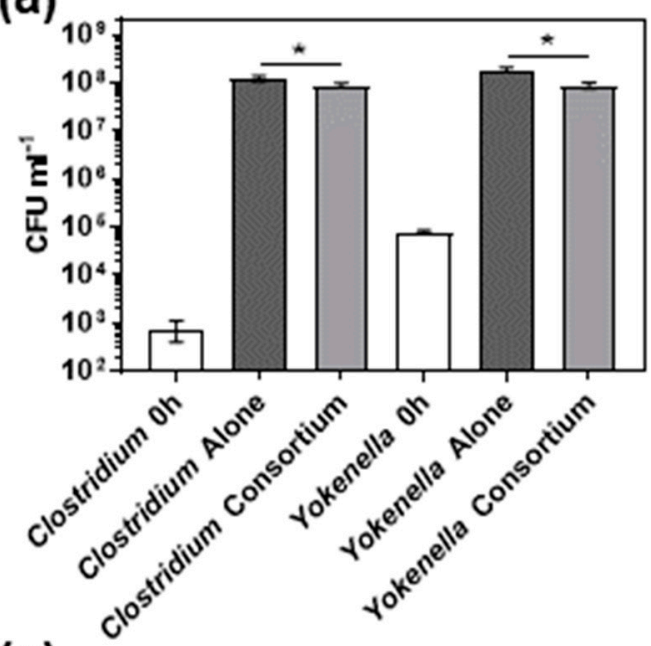

(c)

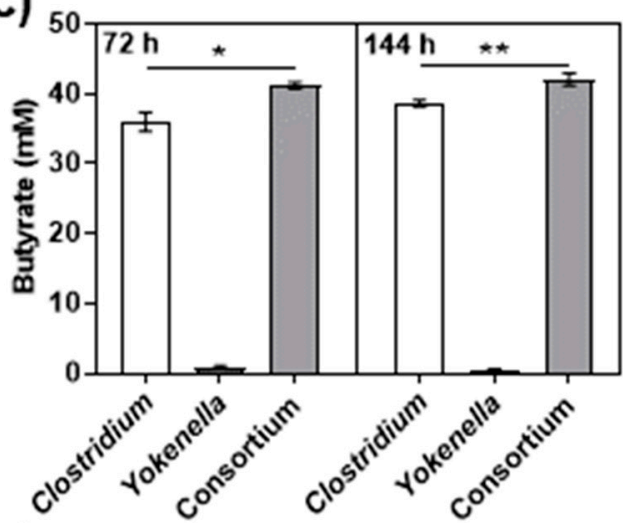

(e)

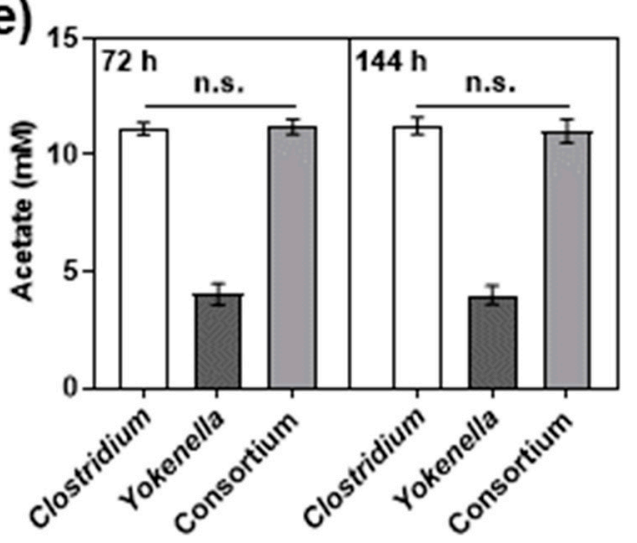

(b)

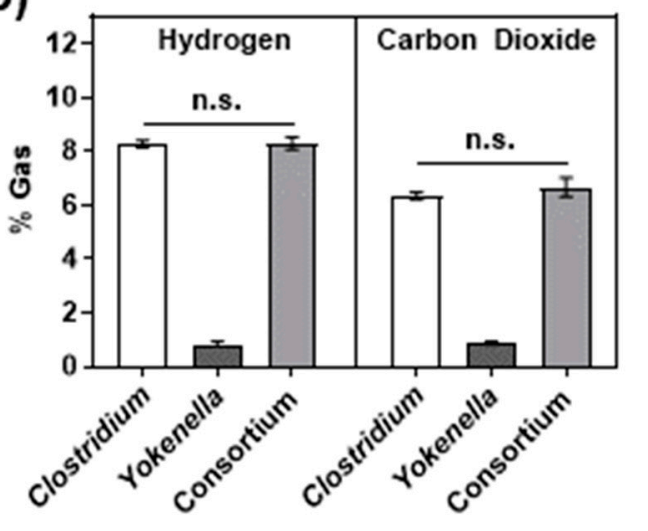

(d)

(f)
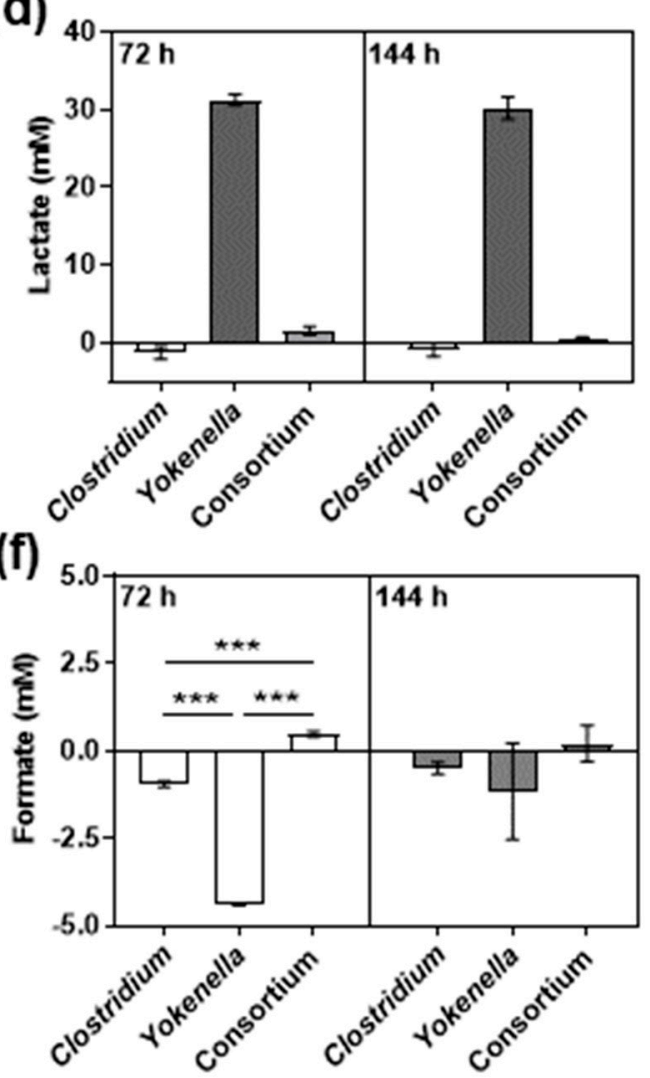

Figure 3. Metabolite production by a consortium of $C$. beijerinckii and Y. regensburgei. Clostridium beijerinckii and Yokenella regensburgei were grown as monocultures or as a consortium on artificial garbage slurry medium (AGS) for $144 \mathrm{~h}$ in an anaerobic chamber, either in gas chromatography vials (b) or sealed tubes $(\mathbf{a}, \mathbf{c}, \mathbf{d}, \mathbf{e})$. (a) Colony forming units after $24 \mathrm{~h}$. (b) Normalized percent hydrogen and carbon dioxide measured by gas chromatography after $72 \mathrm{~h}$. (c) Butyrate, (d) lactate, (e) acetate, and (f) formate production after $72 \mathrm{~h}$ and $144 \mathrm{~h}$. Units for metabolites are $\mathrm{mM}$ as normalized to HPLC standards. The mean and standard error of the mean of four independent replicates are graphed. Asterisks indicate significant difference between the indicated samples (n.s. (not significant), $p>0.05$; ${ }^{*}, p \leq 0.05 ;{ }^{* *}, p \leq 0.01 ; * * *, p \leq 0.001$ by two-tailed Student's $t$ test). 


\subsection{Y. regensburgei Cross-feeds Lactate to C. beijerinckii for Butyrate Production}

We hypothesized that the lactate produced by Y. regensburgei was utilized as a carbon source by C. beijerinckii, which subsequently produced additional butyrate, because a higher concentration of butyrate was observed in the consortium than in the $C$. beijerinckii monoculture (Figure 3c). To examine this possibility in a simpler system, we grew C. beijerinckii or Y. regensburgei on sterile filtered spent media from $24 \mathrm{~h}$ monocultures of $Y$. regensburgei or C. beijerinckii that were grown on Luria Bertani (LB) medium supplemented with $0.5 \%$ glucose. No growth was initially observed of either $C$. beijerinckii or $Y$. regensburgei on the sterile filtered spent media (data not shown); however, addition of sterile $50 \mathrm{mM}$ potassium phosphate $\mathrm{pH} 6.8$ to filtered spent media enabled growth of both strains (Figure 4a), suggesting that decreased media $\mathrm{pH}$ was inhibitory to growth. Growth of both C. beijerinckii and $Y$. regensburgei was significantly greater on spent media derived from the opposing species (i.e., C. beijerinckii growth on Y. regensburgei spent media and Y. regensburgei growth on C. beijerinckii spent media) than observed for growth on spent media derived from the same species (i.e., C. beijerinckii growth on $C$. beijerinckii spent media and $Y$. regensburgei growth on $Y$. regensburgei spent media) (Figure 4a). This is consistent with the idea of cross-feeding between the two species.
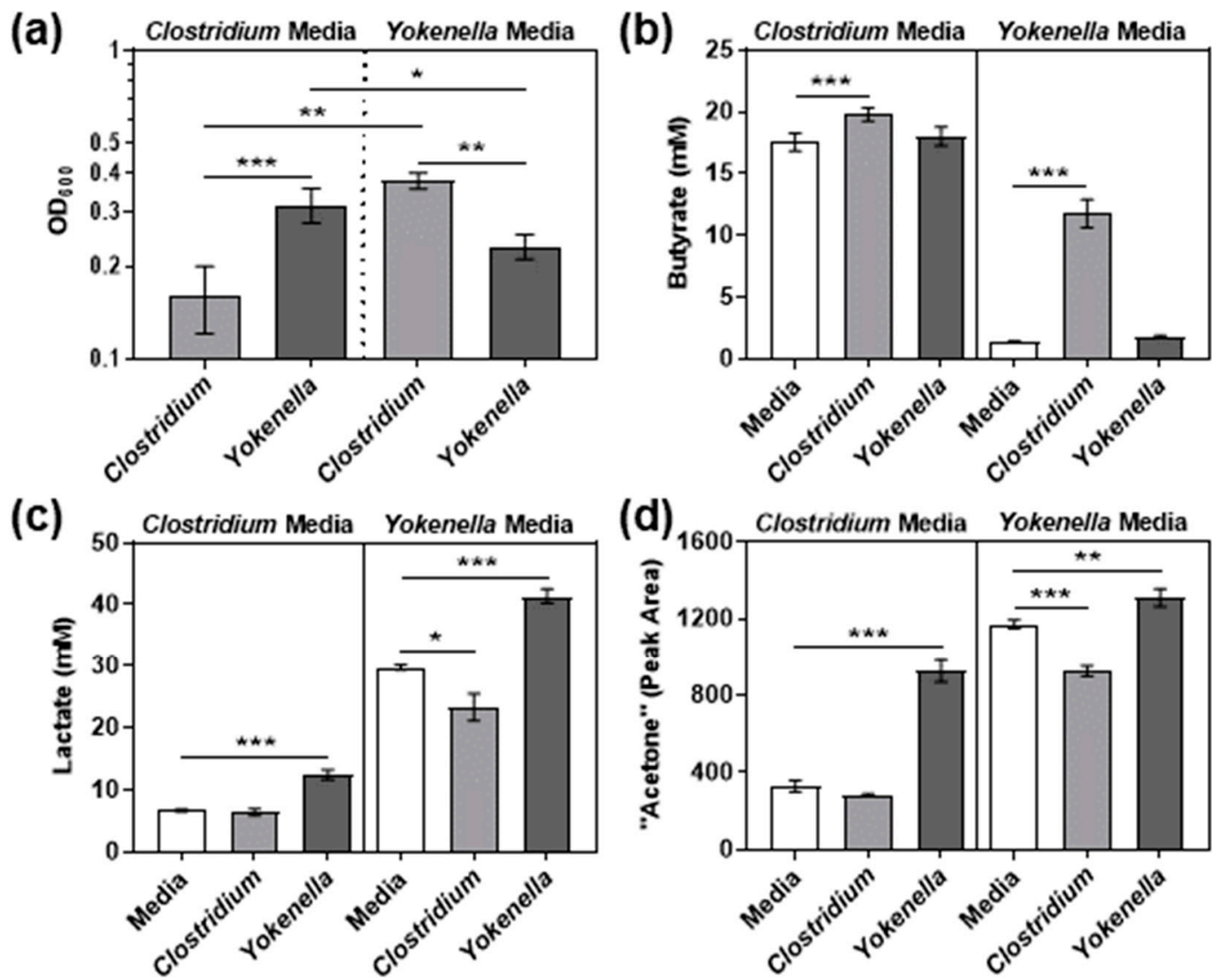

Figure 4. Spent media cross-feeding by C. beijerinckii and Y. regensburgei. Clostridium beijerinckii and Yokenella regensburgei were grown as monocultures for $24 \mathrm{~h}$ on $\mathrm{LB}+0.5 \%$ glucose in an anaerobic chamber. Spent media was sterile filtered and $50 \mathrm{mM}$ potassium phosphate $\mathrm{pH} 6.8$ was added. Spent media from each species was inoculated 1:100 with overnight cultures of either C. beijerinckii or Y. regensburgei and after $24 \mathrm{~h},(\mathbf{a}) \mathrm{OD}_{600}$, (b) butyrate, (c) lactate, and (d) an unknown metabolite that elutes at the expected elution time for acetone, were measured. Units for metabolites are $\mathrm{mM}$ as normalized to HPLC standards. The mean and standard error of the mean of six independent replicates are graphed. Asterisks indicate significant difference between the indicated samples $\left({ }^{*}, p \leq 0.05\right.$; $* *, p \leq 0.01 ;{ }^{* *}, p \leq 0.001$ by two-tailed Student's $t$ test). 
C. beijerinckii significantly increased the concentration of butyrate in spent media from both C. beijerinckii and Y. regensburgei, similar to growth on AGS; however, this increase was substantially larger in the $Y$. regensburgei spent media than the C. beijerinckii spent media ( 700\% versus $\sim 12 \%$ increase) (Figure 4b). C. beijerinckii significantly depletes the lactate from $Y$. regensburgei spent media, concurrent with the large increase in butyrate (Figure 4c), suggesting that lactate produced by Y. regensburgei supports additional growth and butyrate production by C. beijerinckii. Y. regensburgei produces a significant amount of additional lactate in both $C$. beijerinckii and Y. regensburgei spent media (Figure 4c), suggesting that $C$. beijerinckii may produce nutrient(s) capable of being further metabolized by Y. regensburgei, and that $\mathrm{pH}$ is likely a limiting factor for its growth in LB supplemented with glucose.

The HPLC method used for metabolite detection was previously optimized for detection of short-chain fatty acids, acetone, butanol, and ethanol from Clostridium acetobutylicum [51]. A peak eluting at the time of the expected acetone peak was detected in the $Y$. regensburgei spent media (Figure 4d). Y. regensburgei has not previously been reported to produce acetone, and it does not encode the genes necessary to produce it [52]. The C. beijerinckii spent media also contains a metabolite with the same HPLC elution time; however, its peak area is only $\sim 25-30 \%$ of the peak observed in the $Y$. regensburgei spent media. C. beijerinckii significantly depletes the undetermined metabolite when grown on the Y. regensburgei spent media (Figure $4 \mathrm{~d}$ ), while $Y$. regensburgei significantly increased the concentration of the metabolite when grown on both the C. beijerinckii and Y. regensburgei spent media (Figure 4d). Moreover, Y. regensburgei accumulates $\sim 1.7$-fold more of the metabolite when grown anaerobically than aerobically, despite aerobic cultures growing to more than two-fold higher optical density at $600 \mathrm{~nm}$ (data not shown). Electrospray ionization (ESI) mass spectrometry was performed on an HPLC fraction collected from the Y. regensburgei spent media corresponding to the acetone peak. The major mass to charge ratio $(\mathrm{m} / \mathrm{z})$ peaks that were not identified as solvent or carrier peaks were at $\mathrm{m} / \mathrm{z}=171.11$ in the ESI+ spectrum and $\mathrm{m} / \mathrm{z}=127.99$ and 111.00 in the ESI-spectrum (data not shown). These peaks do not map to a known metabolite that could plausibly be produced by $Y$. regensburgei given the current understanding of its metabolism. Further experimentation is required to determine the role of this molecule in potential cross-feeding between Y. regensburgei and C. beijerinckii.

\subsection{C. beijerinckii Uses Lactate as a Carbon Source}

Since C. beijerinckii is capable of depleting lactate from Y. regensburgei spent media (Figure 4c), and the GSMM predicted cross-feeding of lactate from Y. regensburgei to C. beijerinckii (Figure 2), we hypothesized that lactate could support the growth of $C$. beijerinckii either with or without a limited amount of glucose. The optical density of $C$. beijerinckii cultures indeed increased compared to an un-inoculated control in LB media containing $30 \mathrm{mM}$ lactic acid and $20 \mathrm{mM}$ potassium phosphate, when the initial $\mathrm{pH}$ was controlled to 6.7 with sodium hydroxide (Figure $5 \mathrm{a}$ ). Concomitant with the increase in optical density, a significant amount of lactate was depleted from the media, suggesting its use as a carbon source by C. beijerinckii (Figure 5b). Moreover, C. beijerinckii grew (Figure 5a) and significantly depleted lactate from the media (Figure 5b) in cultures with $5 \mathrm{mM}$ glucose and $15 \mathrm{mM}$ lactate within $24 \mathrm{~h}$, although the amount of growth was significantly less than observed in cultures containing $10 \mathrm{mM}$ glucose during the same duration (Figure 5a). In both cases, glucose was completely depleted from the media (data not shown). C. beijerinckii produced primarily butyrate in each of the three conditions, with the amount produced positively correlating with the amount of glucose present (Figure 5c). C. beijerinckii produced substantially more ethanol in the absence of lactate, exhibiting low ethanol production in both the lactate alone and mixed carbon source cultures (Figure 5d). 
(a)

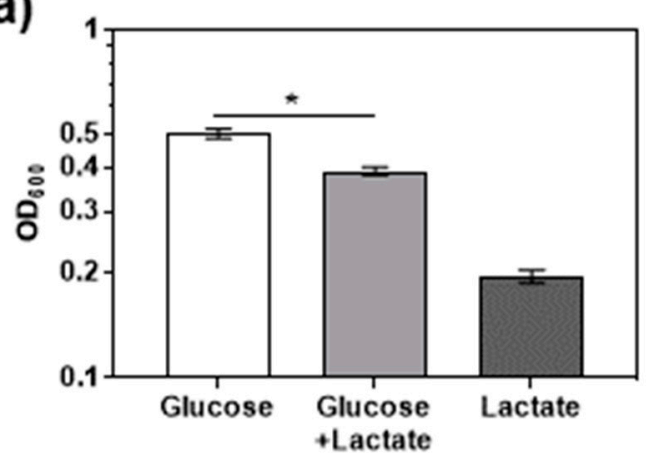

(c)

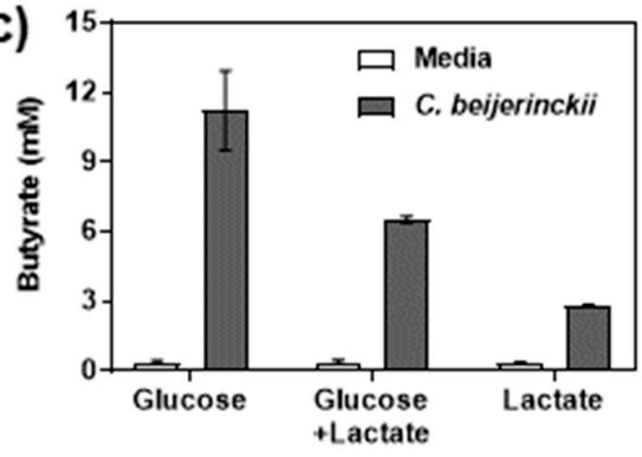

(b)

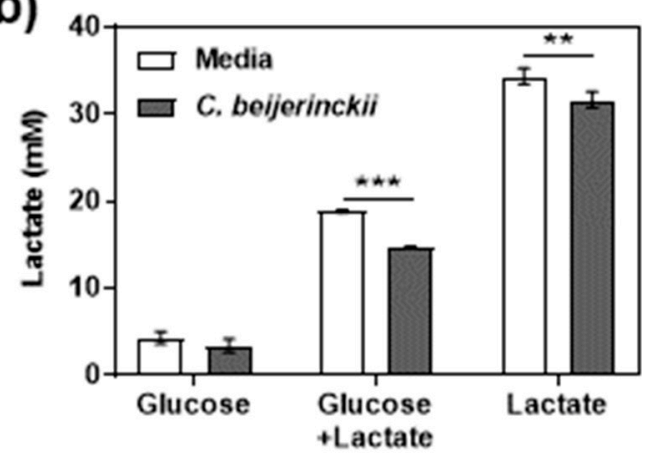

(d)

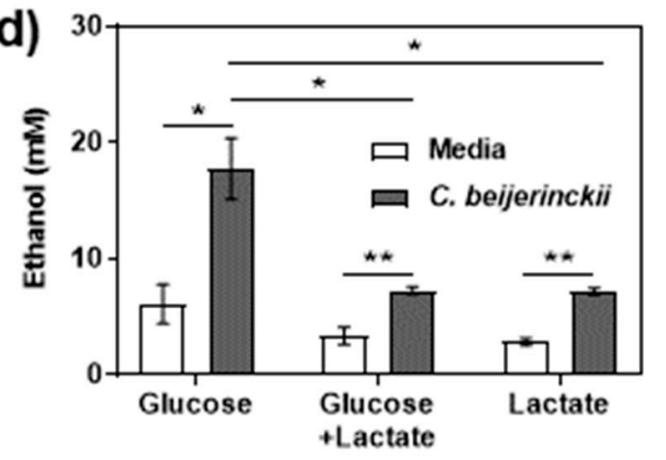

Figure 5. Lactate can support the growth of C. beijerinckii with or without glucose. C. beijerinckii was grown on LB + $20 \mathrm{mM}$ potassium phosphate $\mathrm{pH} 6.8+$ either $10 \mathrm{mM}$ glucose, or $30 \mathrm{mM}$ lactate, or $5 \mathrm{mM}$ glucose and $15 \mathrm{mM}$ lactate, in an anaerobic chamber. (a) $\mathrm{OD}_{600}$, (b) lactate, (c) butyrate, and (d) ethanol, were measured after $24 \mathrm{~h}$ from the C. beijerinckii cultures and un-inoculated controls (Media). Units for metabolites are $\mathrm{mM}$ as normalized to HPLC standards. The mean and standard error of the mean of four independent replicates are graphed. Asterisks indicate significant difference between the indicated samples (n.s., ${ }^{*}, p>0.05 ;{ }^{* *}, p \leq 0.01 ;{ }^{* * *}, p \leq 0.001$ by two-tailed Student's $t$ test).

\subsection{Enhanced Metabolite Production by the Consortium Is Condition-Dependent}

To quantify the production rate and amount of gases produced by C. beijerinckii and Y. regensburgei either alone or as a consortium, we used a DASGIP ${ }^{\circledR}$ parallel bioreactor system coupled with two gas chromatographs to perform near constant measurements of gases (every $11 \mathrm{~min}$ ) from two independent replicates grown in one liter volumes on AGS. The experiment was performed with two distinct sets of bioreactor conditions. First, to closely mimic the closed tubes in the anaerobic chamber, the bioreactor mixtures were left stationary until fifteen seconds prior to sample collection and $\mathrm{pH}$ was not controlled. Second, to maintain the optimal $\mathrm{pH}$ for hydrogen production by the C. beijerinckii hydrogenase [25], the $\mathrm{pH}$ of bioreactor vessels was automatically controlled at $\mathrm{pH}$ 6.7, with continuous stirring at $100 \mathrm{rpm}$ for the entirety of the experiment.

The two bioreactor experiments resulted in distinct patterns of growth and metabolite production for both the consortium and monocultures of $C$. beijerinckii and Y. regensburgei. In the stationary bioreactor experiments without $\mathrm{pH}$ control, $\mathrm{C}$. beijerinckii grew to a higher density in monoculture than the consortium starting eight hours after inoculation and continuing for the duration of the experiment, until it was undetectable in both the monoculture and consortia by the $48 \mathrm{~h}$ time point (Figure 6a). By contrast, no difference was observed in the amount of colony forming units for $Y$. regensburgei between the monoculture and the consortium until the $36 \mathrm{~h}$ time point when there was a 2-log decrease of $Y$. regensburgei in the consortium. Y. regensburgei levels further decreased to undetectable levels by the $48 \mathrm{~h}$ time point (Figure 6a). Although a lower initial amount of C. beijerinckii was present in the consortium, both C. beijerinckii and Y. regensburgei were present in similar amounts from 12-24 $\mathrm{h}$ (Figure 6a). With $\mathrm{pH}$ control, Y. regensburgei maintained a high level of culturable CFUs in both the 
monoculture and the consortium, even at $72 \mathrm{~h}$ (Figure 7a). With $\mathrm{pH}$ control, C. beijerinckii in both monoculture and the consortium reached an apparent peak density at $12 \mathrm{~h}$, before decreasing to nearly undetectable levels by $72 \mathrm{~h}$ (Figure 7a).

C. beijerinckii monocultures and the consortium produced similar amounts of hydrogen (Figure 6b) and carbon dioxide (Figure 6c), without $\mathrm{pH}$ control, though variability between samples was observed. With $\mathrm{pH}$ control, C. beijerinckii monocultures produced greater amounts of hydrogen (Figure $7 \mathrm{~b}$ ) and carbon dioxide (Figure 7c) than the consortium. The total amounts of hydrogen and carbon dioxide produced were higher for fermentations with $\mathrm{pH}$ control than without, although differences in the kinetics between the individual bioreactors of both $\mathrm{C}$. beijerinckii monocultures and the consortium with $\mathrm{pH}$ control were observed. Y. regensburgei produced small amounts of hydrogen (Figures $6 \mathrm{~b}$ and $7 \mathrm{~b}$ ) and carbon dioxide (Figures $6 \mathrm{c}$ and $7 \mathrm{c}$ ) throughout the experiments with and without $\mathrm{pH}$ control.

(a)

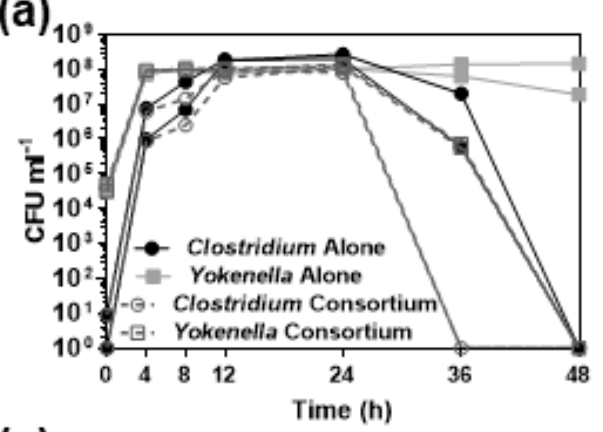

(c)

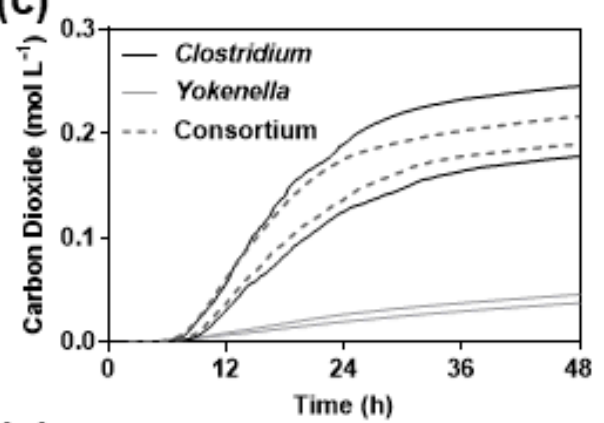

(e)

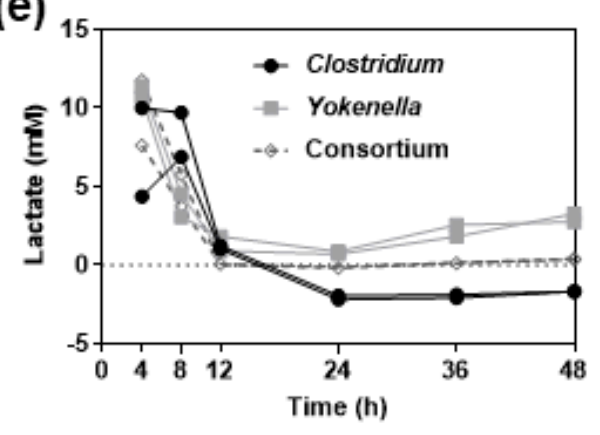

(b)

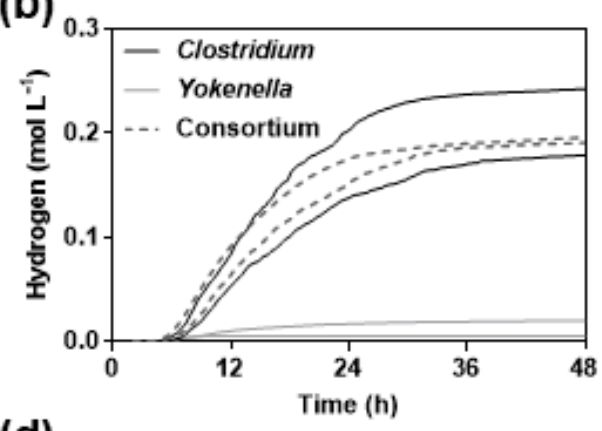

(d)

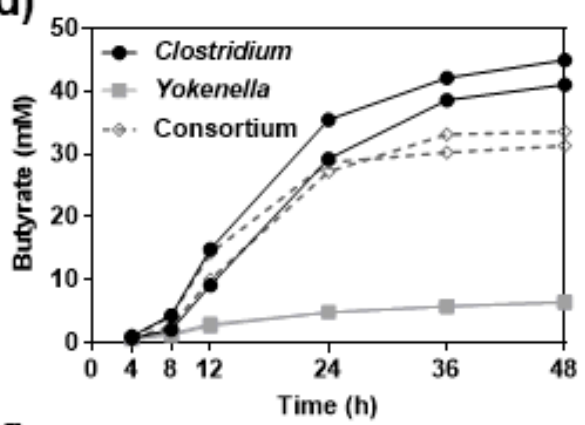

(f)

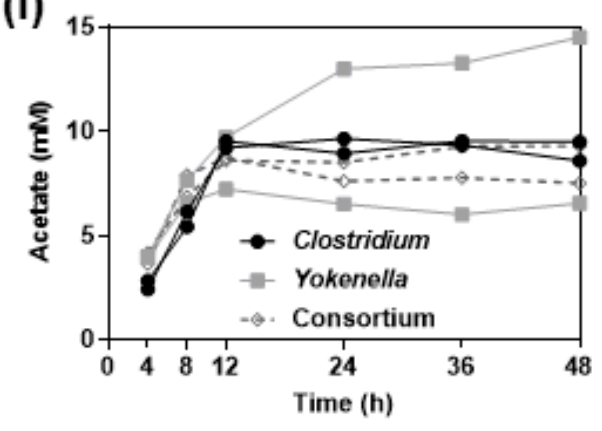

Figure 6. Metabolites produced by the C. beijerinckii and Y. regensburgei consortium in stationary bioreactors. Clostridium beijerinckii and Yokenella regensburgei were grown as monocultures or as a consortium on AGS for $48 \mathrm{~h}$ in $1 \mathrm{~L}$ bioreactor vessels. Vessel contents were only stirred at $100 \mathrm{rpm}$ for $15 \mathrm{~s}$ prior to collection of each sample. (a) Colony forming units, (b) hydrogen, (c) carbon dioxide, (d) butyrate, (e) lactate, (f) acetate quantified at the indicated time points. Each replicate is graphed independently. Units for gas production are moles of gas produced per liter of fermentation volume $\left(\mathrm{mol} \mathrm{L}^{-1}\right)$, derived from the GC gas percentage output using the ideal gas law, headspace flowrate, and the fermentation volume. Units for metabolites are $\mathrm{mM}$ as normalized to HPLC standards. 
(a)

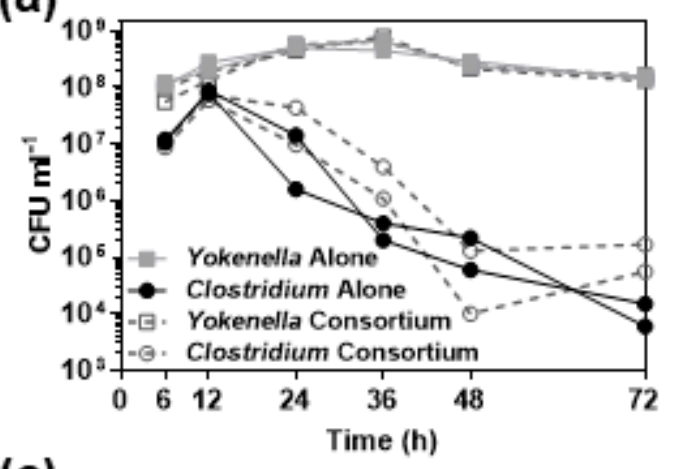

(c)

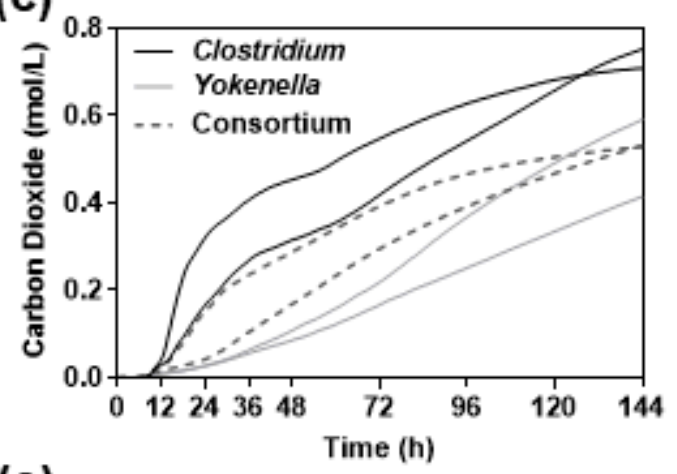

(e)

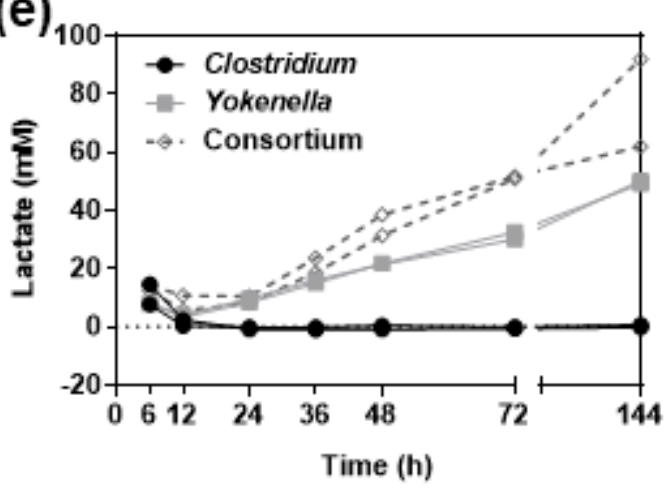

(b)

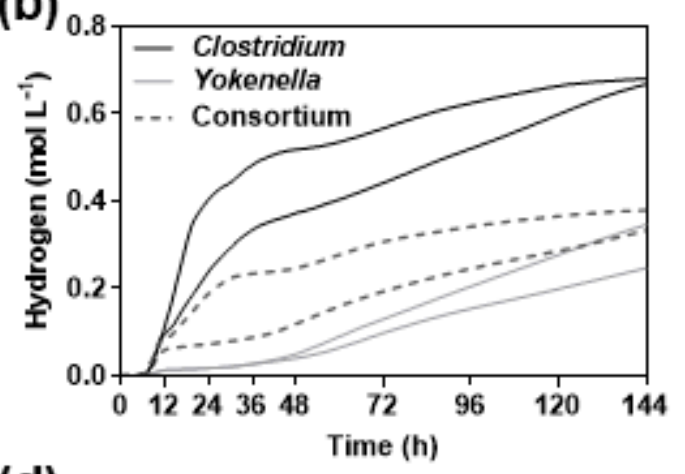

(d)

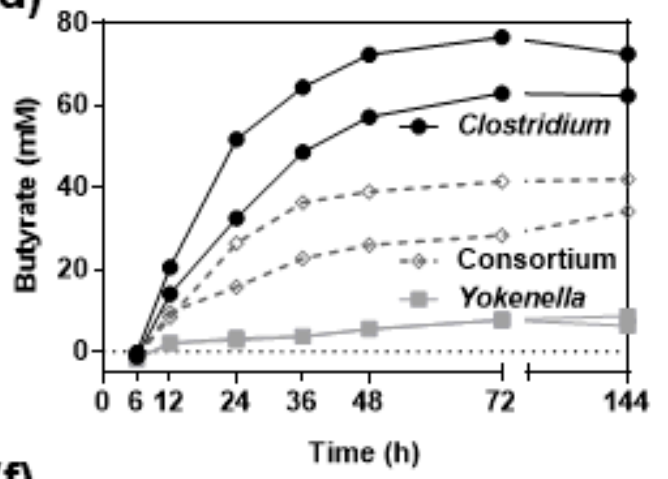

(f)

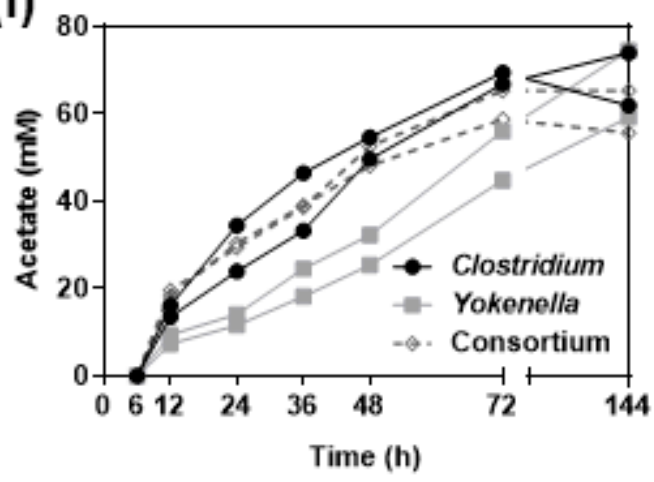

Figure 7. Metabolites produced by the C. beijerinckii and Y. regensburgei consortium in bioreactors with stirring and $\mathrm{pH}$ control. Clostridium beijerinckii and Yokenella regensburgei were grown as monocultures or as a consortium on AGS for $144 \mathrm{~h}$ in $1 \mathrm{~L}$ bioreactor vessels, with the $\mathrm{pH}$ maintained at 6.7 via the addition of sodium hydroxide and constant stirring at $100 \mathrm{rpm}$. (a) Colony forming units, (b) hydrogen, (c) carbon dioxide, (d) butyrate, (e) lactate, (f) acetate quantified at the indicated time points. Each replicate is graphed independently. Units for gas production are moles of gas produced per liter of fermentation volume $\left(\mathrm{mol} \mathrm{L}^{-1}\right)$, derived from the GC gas percentage output using the ideal gas law, headspace flowrate, and the fermentation volume. Units for metabolites are $\mathrm{mM}$ as normalized to HPLC standards.

C. beijerinckii monocultures produced primarily butyrate to a higher level than the consortium both with $\mathrm{pH}$ control (Figure $7 \mathrm{~d}$ ) and without $\mathrm{pH}$ control (Figure $6 \mathrm{~d}$ ). Y. regensburgei monocultures produced primarily lactate in both conditions (Figures 6e and 7e). However, the amount of lactate produced by $Y$. regensburgei with $\mathrm{pH}$ control was lower than the amount of lactate produced by the consortium (Figure 7e), and the amount of lactate produced by $Y$. regensburgei without $\mathrm{pH}$ control was substantially lower than in any other experiment (Figure 6e). C. beijerinckii, Y. regensburgei, and the consortium produced similar levels of acetate (Figures $6 \mathrm{f}$ and $7 \mathrm{f}$ ) and ethanol (data not shown) as secondary fermentation products in both the experiments with and without $\mathrm{pH}$ control. 


\section{Discussion}

Anaerobic fermentation of food waste is an attractive alternative to other disposal methods, to reduce waste volume and recover lost energy [1-3]. Here, we developed a framework to experimentally test metabolic modeling predictions of microbial consortia for their ability to produce commodity chemicals (Figure 1). We have established that a consortium of C. beijerinckii and $Y$. regensburgei is capable of producing increased amounts of commodity chemicals from a simulated food waste medium, compared to monocultures of either species. Genome-scale metabolic modeling predicted that co-cultures of $C$. beijerinckii and $Y$. regensburgei would result in synergistic overproduction of hydrogen as a result of lactate cross-feeding from Y. regensburgei to C. beijerinckii. We observed similar hydrogen gas production by $C$. beijerinckii and the consortium in stationary conditions (Figures $3 b$ and $6 b$ ) and overproduction of the commodity chemicals butyrate (Figure $3 c$ ) and lactate (Figure 7e) by the consortium in distinct experimental conditions. Moreover, we demonstrated that $C$. beijerinckii is capable of using exogenous lactate as a carbon source (Figure 5) and that $Y$. regensburgei cross-feeds lactate to C. beijerinckii (Figure 4). Differences in the metabolites produced by the consortium of $C$. beijerinckii and Y. regensburgei during experiments with the artificial garbage slurry medium (Figures 3,6 and 7) exhibit the difficulty in fermentation scale-up, but also demonstrate that opportunities exist to modulate fermentation conditions for varied commodity chemical output.

\subsection{Experimental Implementation of Genome-Scale Metabolic Modeling Predictions}

The consortium of C. beijerinckii and Y. regensburgei was predicted by GSMM and FBA to have the second highest overproduction of hydrogen gas compared to any individual species from models of 773 gut microbiota species [38,39], leading to its selection for further experimentation. Similar levels of hydrogen were observed for $C$. beijerinckii and the consortium of $C$. beijerinckii and Y. regensburgei in small-scale experiments performed in an anaerobic chamber (Figure 3). This experimental setup prevented quantification of gas from more than a single time point. GC vials from the experiment all had the same headspace volume, but slight variations in growth may have caused different pressures for any given vial at time of headspace analysis. It is possible to measure the pressure in a given vial; however, this was not done, as it is difficult to obtain a pressure measurement without releasing gas from the GC vial, which could potentially affect the GC concentration measurements. Continuous GC monitoring over time was performed from $1 \mathrm{~L}$ bioreactor cultures (Figures 6 and 7). For stationary cultures without $\mathrm{pH}$ control, which most closely mimicked the small-scale experimental design, the hydrogen production by the consortium was similar to the amount of hydrogen produced by C. beijerinckii monocultures (Figure 6b). While the consortium did not produce similar amounts of hydrogen to $C$. beijerinckii monocultures grown with $\mathrm{pH}$ control, which exhibited the highest amounts of hydrogen produced from any experimental condition tested (Figure 7b), the FBA modeling did not account for such conditions. Manipulating simulated conditions with FBA modeling could provide additional avenues to improve commodity chemical production.

\subsection{Y. regensburgei Cross-feeding to C. beijerinckii}

In small-scale fermentations of the AGS, substantially lower lactate was produced by the consortium than would be expected if the $Y$. regensburgei present produced a proportional amount of lactate to that in the Y. regensburgei monoculture (Figure $3 \mathrm{~d}$ ). This suggested that the predicted cross-feeding of lactate from Y. regensburgei to $C$. beijerinckii (Figure 2) may be occurring when the consortium was grown in AGS. Clostridium butyricum [53] and Clostridium saccharoperbutylacetonicum [54] were previously described to metabolize acetate and lactate to butyrate, while several other Clostridium, including C. beijerinckii, were proposed to breakdown lactate [55]. Here, we show that C. beijerinckii can metabolize a limited amount of lactate, with or without a limited amount of glucose (Figure 5). The proposed mechanism for lactate metabolism in Clostridium is based on the lactate oxidation pathway in Acetobacterium woodii, which couples a flavin adenine dinucleotide (FAD)-dependent lactate 
dehydrogenase with an electron flavoprotein complex to convert a reduced ferredoxin, lactate, and two oxidized nicotinamide adenine dinucleotides (NAD) to an oxidized ferredoxin, pyruvate, and two reduced nicotinamide adenine dinucleotides (NADH) [53]. A similar mechanism is likely used by $C$. beijerinckii, as a genomic locus with significant sequence homology to the locus proposed to breakdown lactate in C. butyricum [53] is found in C. beijerinckii (Cbei_2884-Cbei_2888) using the Basic Local Alignment Search Tool (BLAST) [56]. While FBA predicts that multiple species can cross-feed lactate to C. beijerinckii, the ability to cross-feeding of lactate to Clostridium may be somewhat limited, as lactic acid bacteria, which produce large amounts of lactate, are often detrimental to efforts to produce hydrogen from complex microbial consortia [55]. Moreover, a pilot experiment attempting to cross-feed lactate from the lactic acid bacterium Lactobacillus fermentum to C. beijerinckii was unsuccessful, as L. fermentum produced a significant amount of lactate $(\sim 30 \mathrm{mM})$, but no change in lactate or butyrate levels was observed when $C$. beijerinckii was cultured in the spent medium (data not shown).

\subsection{Varying Growth Conditions to Control Metabolic Output}

While small variability in metabolic output was observed between replicates with small-scale cultures (Figure 3), a substantial amount of variability was observed between all individual replicates containing C. beijerinckii for the bioreactor experiments (Figures 6 and 7). Part of this variability may stem from differences in handling of the inoculums for the bioreactors versus the small-scale cultures. C. beijerinckii is sensitive to oxygen, and inoculation of the bioreactors required brief exposure to oxygen as the inoculum was transported from an anaerobic chamber to the anaerobic environment of the bioreactors. This exposure coupled with slight differences in the growth phase or culture density of the $C$. beijerinckii inocula could have biased individual bioreactors to be more or less favorable for C. beijerinckii growth. The AGS is another potential source of variability, as the precise composition of the main component could have varied from vessel to vessel. The $\mathrm{pH}$ control also could have introduced variability between replicates, because noticeably different amounts of sodium hydroxide were automatically added to each vessel, which differentially affected the $\mathrm{pH}$ and osmolality of the medium. Both $\mathrm{pH}$ and sodium concentration can have profound effects on Clostridium metabolic output $[57,58]$.

In the experiments with $\mathrm{pH}$ control, the consortium of $C$. beijerinckii and $Y$. regensburgei produced a drastically different metabolic profile than in other experiments (Figure 7). After an initial lactate decrease, which corresponded to an increase in C. beijerinckii colony forming units (Figures 7a and 6e), the consortium cultures produced more lactate than $Y$. regensburgei monocultures. This increase in lactate corresponded to a decrease in C. beijerinckii, while Y. regensburgei levels remained near constant. Thus, Y. regensburgei likely dominated the metabolite profile of the cultures, and C. beijerinckii may have either shifted its metabolism to produce additional lactate or cross-fed a metabolite to Y. regensburgei increasing its lactate production. Modifying the metabolic output of a consortium by either controlling or not controlling the culture $\mathrm{pH}$ is a mechanism that could be used to increase the agility of a designed microbial consortium for a future application.

\subsection{Microbial Consortia with Distinct Mechanisms of Chemical Overproduction}

Modeling predicted the consortium of $C$. beijerinckii and $Y$. regensburgei to produce increased $C$. beijerinckii biomass (Table 1), due to the cross-feeding of lactate from Y. regensburgei to C. beijerinckii that was experimentally observed (Figure 4). Other consortia that were predicted to produce an increased amount of hydrogen compared to C. beijerinckii monocultures (Table 1) likely have different mechanisms that could contribute to commodity chemical overproduction. For example, Cellulosimicrobium cellulans can break down cellulose and xylans [59], complex polysaccharides common in plant material [60], which are inaccessible to C. beijerinckii. In contrast to the consortium of C. beijerinckii and Y. regensburgei, FBA predicted that the biomass of both C. beijerinckii and Cellulosimicrobium cellulans would increase in a co-culture compared to monocultures (Table 1), perhaps due to increased available carbon and cross-feeding of metabolites from C. beijerinckii to Cellulsimicrobium cellulans. Similarly, members 
of the Capnocytophaga genus can metabolize complex polysaccharides [61] and carbon dioxide [62], which could explain the predicted hydrogen overproduction by a consortium of $C$. beijerinckii and Capnocytophaga sputigena (Table 1). Integrating genome-scale metabolic model improvements and testing additional consortia with different predicted interactions could enable discovery of pathways to enhanced commodity chemical production.

\section{Conclusions}

Genome-scale metabolic models and flux-balance analysis predicted several microbial consortia expected to produce a significantly greater amount of hydrogen than any individual species [39] (Table 1). We focused on the consortium of $C$. beijerinckii and Y. regensburgei that was predicted to have the greatest increase in biomass and hydrogen production by C. beijerinckii. The flux-balance analysis predicted the increase in hydrogen and biomass based on the cross-feeding of lactate to C. beijerinckii (Figure 2). We established that cross-feeding of lactate from Y. regensburgei to C. beijerinckii can occur (Figure 4), and that exogenous lactate is capable of supporting the growth of C. beijerinckii (Figure 5). We were unable to demonstrate that growing C. beijerinckii as part of a consortium with Y. regensburgei increased the production of hydrogen above the levels observed for $C$. beijerinckii monocultures (Figure 3). The consortium was capable of producing more butyrate (Figure 3c) or lactate (Figure 7e) than individual monocultures, depending on the growth conditions. Examining the effects of adding another species that is predicted to have a different role in promoting hydrogen production than cross-feeding of lactate to the consortium of C. beijerinckii and Y. regensburgei, or testing that species with $C$. beijerinckii alone could provide better improvement of hydrogen production from the artificial garbage slurry than was observed. The framework presented here can be used to screen large numbers of possible microbial combinations by first using genome-scale metabolic modeling and flux-balance analysis to predict consortia with an increased likelihood to convert waste to commodity chemicals and then testing the highest producing consortia experimentally for their ability to produce chemicals of interest from readily available materials.

Author Contributions: N.D.S., M.A.P., and K.L.A. conceptualized the research; M.A.P. performed the GSMM and FBA, and wrote the original draft of the Introduction, Materials and Methods, and Results section for the GSMM. W.M. performed the HPLC and GC analysis and wrote the original draft of the Materials and Methods section for the HPLC and GC. N.D.S. and E.S.G. performed the bioreactor fermentation experiments and edited the paper. M.B. performed the analysis of the bioreactor GC data. N.D.S. performed all other experiments and wrote the original draft of the remainder of the paper.

Funding: This research received no external funding.

Acknowledgments: The authors would like to acknowledge members of the Combat Capabilities and Development Command Army Research Laboratory for useful discussions and Yue Li of the University of Maryland for performing mass spectrometry.

Conflicts of Interest: The authors declare no conflict of interest. The funders had no role in the design of the study; in the collection, analyses, or interpretation of data; in the writing of the manuscript, or in the decision to publish the results.

\section{References}

1. Xu, F.; Li, Y.; Ge, X.; Yang, L.; Li, Y. Anaerobic digestion of food waste-Challenges and opportunities. Bioresour. Technol. 2018, 247, 1047-1058. [CrossRef] [PubMed]

2. Ren, Y.; Yu, M.; Wu, C.; Wang, Q.; Gao, M.; Huang, Q.; Liu, Y. A comprehensive review on food waste anaerobic digestion: Research updates and tendencies. Bioresour. Technol. 2018, 247, 1069-1076. [CrossRef] [PubMed]

3. Ma, Y.; Liu, Y. Turning food waste to energy and resources towards a great environmental and economic sustainability: An innovative integrated biological approach. Biotechnol. Adv. 2019. [CrossRef] [PubMed]

4. Rock, K.; Lesher, L.; Kramer, F.M.; Johnson, J.; Bordic, M.; Miller, H. An Analysis of Military Field Feeding Waste. In U. S. Army Natick Soldier Systems Center Technology Report Natick/Tr-00/021; U.S. Army Natick Soldier Systems Center: Natick, MA, USA, 2000. 
5. Knowlton, L.; Pickard, D.; Diebold, J.; Lasnik, L.; Lilley, A.; Browne, K. On-Site Field-Feeding Waste to Energy Converter. 2008. Available online: http://nsrdeec.natick.army.mil/LIBRARY/00-09/R08-105.pdf (accessed on 15 August 2019).

6. Birgen, C.; Durre, P.; Preisig, H.A.; Wentzel, A. Butanol production from lignocellulosic biomass: Revisiting fermentation performance indicators with exploratory data analysis. Biotechnol. Biofuels 2019, 12, 167. [CrossRef] [PubMed]

7. Cairns, T.C.; Zheng, X.; Zheng, P.; Sun, J.; Meyer, V. Moulding the mould: Understanding and reprogramming filamentous fungal growth and morphogenesis for next generation cell factories. Biotechnol. Biofuels 2019, 12, 77. [CrossRef]

8. Shin, H.D.; McClendon, S.; Vo, T.; Chen, R.R. Escherichia coli binary culture engineered for direct fermentation of hemicellulose to a biofuel. Appl. Environ. Microbiol. 2010, 76, 8150-8159. [CrossRef]

9. Zhang, H.; Pereira, B.; Li, Z.; Stephanopoulos, G. Engineering Escherichia coli coculture systems for the production of biochemical products. Proc. Natl. Acad. Sci. USA 2015, 112, 8266-8271. [CrossRef]

10. Salmela, M.; Lehtinen, T.; Efimova, E.; Santala, S.; Mangayil, R. Metabolic pairing of aerobic and anaerobic production in a one-pot batch cultivation. Biotechnol. Biofuels 2018, 11, 187. [CrossRef]

11. Kim, H.; Jeon, B.S.; Pandey, A.; Sang, B.I. New coculture system of Clostridium spp. and Megasphaera hexanoica using submerged hollow-fiber membrane bioreactors for caproic acid production. Bioresour. Technol. 2018, 270, 498-503. [CrossRef]

12. Wen, Z.; Minton, N.P.; Zhang, Y.; Li, Q.; Liu, J.; Jiang, Y.; Yang, S. Enhanced solvent production by metabolic engineering of a twin-clostridial consortium. Metab. Eng. 2017, 39, 38-48. [CrossRef]

13. Gomez-Flores, M.; Nakhla, G.; Hafez, H. Hydrogen production and microbial kinetics of Clostridium termitidis in mono-culture and co-culture with Clostridium beijerinckii on cellulose. AMB Express 2017, 7, 84. [CrossRef] [PubMed]

14. Roell, G.W.; Zha, J.; Carr, R.R.; Koffas, M.A.; Fong, S.S.; Tang, Y.J. Engineering microbial consortia by division of labor. Microb. Cell Fact. 2019, 18, 35. [CrossRef]

15. Zhou, K.; Qiao, K.; Edgar, S.; Stephanopoulos, G. Distributing a metabolic pathway among a microbial consortium enhances production of natural products. Nat. Biotechnol. 2015, 33, 377-383. [CrossRef] [PubMed]

16. Cavaliere, M.; Feng, S.; Soyer, O.S.; Jimenez, J.I. Cooperation in microbial communities and their biotechnological applications. Environ. Microbiol. 2017, 19, 2949-2963. [CrossRef] [PubMed]

17. LaSarre, B.; McCully, A.L.; Lennon, J.T.; McKinlay, J.B. Microbial mutualism dynamics governed by dose-dependent toxicity of cross-fed nutrients. ISME J. 2017, 11, 337-348. [CrossRef]

18. Charubin, K.; Papoutsakis, E.T. Direct cell-to-cell exchange of matter in a synthetic Clostridium syntrophy enables CO2 fixation, superior metabolite yields, and an expanded metabolic space. Metab. Eng. 2019, 52, 9-19. [CrossRef]

19. Benomar, S.; Ranava, D.; Cardenas, M.L.; Trably, E.; Rafrafi, Y.; Ducret, A.; Hamelin, J.; Lojou, E.; Steyer, J.P.; Giudici-Orticoni, M.T. Nutritional stress induces exchange of cell material and energetic coupling between bacterial species. Nat. Commun. 2015, 6, 7283. [CrossRef]

20. Kosina, S.M.; Danielewicz, M.A.; Mohammed, M.; Ray, J.; Suh, Y.; Yilmaz, S.; Singh, A.K.; Arkin, A.P.; Deutschbauer, A.M.; Northen, T.R. Exometabolomics Assisted Design and Validation of Synthetic Obligate Mutualism. ACS Synth. Biol. 2016, 5, 569-576. [CrossRef]

21. Smith, N.W.; Shorten, P.R.; Altermann, E.; Roy, N.C.; McNabb, W.C. The Classification and Evolution of Bacterial Cross-Feeding. Front. Ecol. Evol. 2019, 7. [CrossRef]

22. Łukajtis, R.; Hołowacz, I.; Kucharska, K.; Glinka, M.; Rybarczyk, P.; Przyjazny, A.; Kamiński, M. Hydrogen production from biomass using dark fermentation. Renew. Sustain. Energy Rev. 2018, 91, 665-694. [CrossRef]

23. Keskin, T.; Abo-Hashesh, M.; Hallenbeck, P.C. Photofermentative hydrogen production from wastes. Bioresour. Technol. 2011, 102, 8557-8568. [CrossRef] [PubMed]

24. Yoo, M.; Bestel-Corre, G.; Croux, C.; Riviere, A.; Meynial-Salles, I.; Soucaille, P. A Quantitative System-Scale Characterization of the Metabolism of Clostridium Acetobutylicum. mBio 2015, 6, 1808-1815. [CrossRef] [PubMed]

25. Masset, J.; Calusinska, M.; Hamilton, C.; Hiligsmann, S.; Joris, B.; Wilmotte, A.; Thonart, P. Fermentative hydrogen production from glucose and starch using pure strains and artificial co-cultures of Clostridium Spp. Biotechnol. Biofuels 2012, 5, 35. [CrossRef] [PubMed] 
26. Liu, B.F.; Ren, N.Q.; Xie, G.J.; Ding, J.; Guo, W.Q.; Xing, D.F. Enhanced bio-hydrogen production by the combination of dark- and photo-fermentation in batch culture. Bioresour. Technol. 2010, 101, 5325-5329. [CrossRef] [PubMed]

27. Laurinavichene, T.; Laurinavichius, K.; Shastik, E.; Tsygankov, A. Long-term $\mathrm{H}_{2}$ photoproduction from starch by co-culture of Clostridium butyricum and Rhodobacter sphaeroides in a repeated batch process. Biotechnol. Lett. 2018, 40, 309-314. [CrossRef]

28. Nasr, N.; Gupta, M.; Hafez, H.; El Naggar, M.H.; Nakhla, G. Mono- and co-substrate utilization kinetics using mono- and co-culture of Clostridium beijerinckii and Clostridium saccharoperbutylacetonicum. Bioresour. Technol. 2017, 241, 152-160. [CrossRef]

29. Maeda, T.; Tran, K.T.; Yamasaki, R.; Wood, T.K. Current state and perspectives in hydrogen production by Escherichia coli: Roles of hydrogenases in glucose or glycerol metabolism. Appl. Microbiol. Biotechnol. 2018, 102, 2041-2050. [CrossRef]

30. Seppälä, J.J.; Puhakka, J.A.; Yli-Harja, O.; Karp, M.T.; Santala, V. Fermentative hydrogen production by Clostridium butyricum and Escherichia coli in pure and cocultures. Int. J. Hydrogen Energy 2011, 36, 10701-10708. [CrossRef]

31. Schellenberger, J.; Que, R.; Fleming, R.M.; Thiele, I.; Orth, J.D.; Feist, A.M.; Zielinski, D.C.; Bordbar, A.; Lewis, N.E.; Rahmanian, S.; et al. Quantitative prediction of cellular metabolism with constraint-based models: The COBRA Toolbox v2.0. Nat. Protoc. 2011, 6, 1290-1307. [CrossRef]

32. Orth, J.D.; Thiele, I.; Palsson, B.O. What is flux balance analysis? Nat. Biotechnol. 2010, 28, 245-248. [CrossRef]

33. McCloskey, D.; Palsson, B.O.; Feist, A.M. Basic and applied uses of genome-scale metabolic network reconstructions of Escherichia coli. Mol. Syst. Biol. 2013, 9, 661. [CrossRef] [PubMed]

34. Zhuang, K.; Izallalen, M.; Mouser, P.; Richter, H.; Risso, C.; Mahadevan, R.; Lovley, D.R. Genome-scale dynamic modeling of the competition between Rhodoferax and Geobacter in anoxic subsurface environments. ISME J. 2011, 5, 305-316. [CrossRef] [PubMed]

35. Stolyar, S.; Van Dien, S.; Hillesland, K.L.; Pinel, N.; Lie, T.J.; Leigh, J.A.; Stahl, D.A. Metabolic modeling of a mutualistic microbial community. Mol. Syst. Biol. 2007, 3, 92. [CrossRef] [PubMed]

36. Bauer, E.; Zimmermann, J.; Baldini, F.; Thiele, I.; Kaleta, C. BacArena: Individual-based metabolic modeling of heterogeneous microbes in complex communities. PLoS Comput. Biol. 2017, 13, e1005544. [CrossRef] [PubMed]

37. Harcombe, W.R.; Riehl, W.J.; Dukovski, I.; Granger, B.R.; Betts, A.; Lang, A.H.; Bonilla, G.; Kar, A.; Leiby, N.; Mehta, P.; et al. Metabolic resource allocation in individual microbes determines ecosystem interactions and spatial dynamics. Cell Rep. 2014, 7, 1104-1115. [CrossRef] [PubMed]

38. Magnusdottir, S.; Heinken, A.; Kutt, L.; Ravcheev, D.A.; Bauer, E.; Noronha, A.; Greenhalgh, K.; Jager, C.; Baginska, J.; Wilmes, P.; et al. Generation of genome-scale metabolic reconstructions for 773 members of the human gut microbiota. Nat. Biotechnol. 2016. [CrossRef]

39. Perisin, M.A.; Sund, C.J. Human gut microbe co-cultures have greater potential than monocultures for food waste remediation to commodity chemicals. Sci. Rep. 2018, 8, 15594. [CrossRef]

40. R Core Team. R: A language and environment for statistical computing. In $R$ Foundation for Statistical Computing; R Core Team: Vienna, Austria, 2015; Available online: http://www.R-project.org/ (accessed on 24 September 2019).

41. Wickham, H. Ggplot2: Elegant Graphics for Data Analysis; Springer: New York, NY, USA, 2009.

42. Fritzemeier, C.J.; Gelius-Dietrich, G.; Alzoubi, D.; Habil, A. sybilSBML: SBML Integration in Package 'Sybil'. 2017. Available online: https://cran.r-project.org/web/packages/sybilSBML/index.html (accessed on 24 September 2019).

43. Gelius-Dietrich, G.; Desouki, A.A.; Fritzemeier, C.J.; Lercher, M.J. Sybil-Efficient constraint-based modelling in R. BMC Syst. Biol. 2013, 7, 125. [CrossRef]

44. Fritzemeier, C.J.; Gelius-Dietrich, G.; Luangkesorn, L. glpkAPI: R Interface to C API of GLPK. 2015. Available online: https://cran.r-project.org/web/packages/glpkAPI/index.html (accessed on 24 September 2019).

45. Heirendt, L.; Arreckx, S.; Pfau, T.; Mendoza, S.N.; Richelle, A.; Heinken, A.; Haraldsdottir, H.S.; Keating, S.M.; Vlasov, V.; Wachowiak, J.; et al. Creation and analysis of biochemical constraint-based models: The COBRA Toolbox v3.0. Nat. Protoc 2019, 14, 639-702. [CrossRef]

46. Klitgord, N.; Segre, D. Environments that Induce Synthetic Microbial Ecosystems. PLoS Comput. Biol. 2010, 6, 1001002. [CrossRef] 
47. Roos, J.W.; McLaughlin, J.K.; Papoutsakis, E.T. The effect of pH on nitrogen supply, cell lysis, and solvent production in fermentations of Clostridium acetobutylicum. Biotechnol. Bioeng. 1985, 27, 681-694. [CrossRef] [PubMed]

48. O'Brien, R.W.; Morris, J.G. The Ferredoxin-dependent reduction of chloramphenicol by clostridium acetobutylicum. J. Gen. Microbiol. 1971, 67, 265-271. [CrossRef] [PubMed]

49. Stock, I.; Sherwood, K.J.; Wiedemann, B. Antimicrobial susceptibility patterns, beta-lactamases, and biochemical identification of Yokenella regensburgei strains. Diagn. Microbiol. Infect. Dis. 2004, 48, 5-15. [CrossRef] [PubMed]

50. Sasaki, K.; Morita, M.; Hirano, S.; Ohmura, N.; Igarashi, Y. Effect of adding carbon fiber textiles to methanogenic bioreactors used to treat an artificial garbage slurry. J. Biosci. Bioeng. 2009, 108, 130-135. [CrossRef] [PubMed]

51. Finch, A.S.; Mackie, T.D.; Sund, C.J.; Sumner, J.J. Metabolite analysis of Clostridium acetobutylicum: Fermentation in a microbial fuel cell. Bioresour. Technol. 2011, 102, 312-315. [CrossRef] [PubMed]

52. Kosako, Y.; Sakazaki, R.; Yoshizaki, E. Yokenella regensburgei gen. nov., sp. nov.: A new genus and species in the family Enterobacteriaceae. Jpn. J. Med. Sci. Biol. 1984, 37, 117-124. [CrossRef]

53. Detman, A.; Mielecki, D.; Chojnacka, A.; Salamon, A.; Błaszczyk, M.K.; Sikora, A. Cell factories converting lactate and acetate to butyrate: Clostridium butyricum and microbial communities from dark fermentation bioreactors. Microb. Cell Fact. 2019, 18. [CrossRef]

54. Yoshida, T.; Tashiro, Y.; Sonomoto, K. Novel high butanol production from lactic acid and pentose by Clostridium Saccharoperbutylacetonicum. J. Biosci. Bioeng. 2012, 114, 526-530. [CrossRef]

55. Cabrol, L.; Marone, A.; Tapia-Venegas, E.; Steyer, J.-P.; Ruiz-Filippi, G.; Trably, E. Microbial ecology of fermentative hydrogen producing bioprocesses: Useful insights for driving the ecosystem function. FEMS Microbiol. Rev. 2017, 41, 158-181. [CrossRef]

56. Altschul, S.F.; Gish, W.; Miller, W.; Myers, E.W.; Lipman, D.J. Basic local alignment search tool. J. Mol. Biol. 1990, 215, 403-410. [CrossRef]

57. Zhao, X.; Condruz, S.; Chen, J.; Jolicoeur, M. A quantitative metabolomics study of high sodium response in Clostridium acetobutylicum ATCC 824 acetone-butanol-ethanol (ABE) fermentation. Sci. Rep. 2016, 6. [CrossRef] [PubMed]

58. Patakova, P.; Branska, B.; Sedlar, K.; Vasylkivska, M.; Jureckova, K.; Kolek, J.; Koscova, P.; Provaznik, I. Acidogenesis, solventogenesis, metabolic stress response and life cycle changes in Clostridium beijerinckii NRRL B-598 at the transcriptomic level. Sci. Rep. 2019, 9. [CrossRef] [PubMed]

59. Dou, T.-Y.; Chen, J.; Hao, Y.-F.; Qi, X. Effects of Different Carbon Sources on Enzyme Production and Ultrastructure of Cellulosimicrobium cellulans. Curr. Microbiol. 2019, 76, 355-360. [CrossRef] [PubMed]

60. Wierzbicki, M.P.; Maloney, V.; Mizrachi, E.; Myburg, A.A. Xylan in the Middle: Understanding Xylan Biosynthesis and Its Metabolic Dependencies Toward Improving Wood Fiber for Industrial Processing. Front. Plant. Sci. 2019, 10. [CrossRef] [PubMed]

61. Manfredi, P.; Renzi, F.; Mally, M.; Sauteur, L.; Schmaler, M.; Moes, S.; Jenö, P.; Cornelis, G.R. The genome and surface proteome of Capnocytophaga canimorsus reveal a key role of glycan foraging systems in host glycoproteins deglycosylation. Mol. Microbiol. 2011, 81, 1050-1060. [CrossRef]

62. Kapke, P.A.; Brown, A.T.; Lillich, T.T. Carbon dioxide metabolism by Capnocytophaga ochracea: Identification, characterization, and regulation of a phosphoenolpyruvate carboxykinase. Infect. Immun. 1980, 27, 756-766.

(C) 2019 by the authors. Licensee MDPI, Basel, Switzerland. This article is an open access article distributed under the terms and conditions of the Creative Commons Attribution (CC BY) license (http://creativecommons.org/licenses/by/4.0/). 